\title{
Personalized 3D Printed Ciprofloxacin Impregnated Meshes for the Management of Hernia
}

\author{
Nadia Qamar ${ }^{\text {a }}$, Nasir Abbas ${ }^{\text {a }}$, Muhammad Irfan ${ }^{\text {b }}$, Amjad Hussain ${ }^{\text {a }}$, Muhammad Sohail \\ Arshad ${ }^{\mathrm{c}}$, Sumera Latif a, Faisal Mehmood ${ }^{\mathrm{a}}$, Muhammad Usman Ghori ${ }^{\mathrm{d}}$.
}

${ }^{a}$ University College of Pharmacy, University of the Punjab, Lahore, Pakistan

${ }^{b}$ Department of Pharmacy, Government College University, Faisalabad, Pakistan

${ }^{c}$ Department of Pharmacy, Bahaudin Zakaria University, Multan, Pakistan

${ }^{d}$ Department of Pharmacy, University of Huddersfield, Huddersfield, UK

*Corresponding author. University College of Pharmacy, University of the Punjab, Lahore Pakistan

Tel: +923317724909, +924299211616

nasirabbas77@gmail.com 


\section{Abstract:}

This study aims to explore the application of fused deposition modeling (FDM), a widely used 3D printing technique, in the fabrication of personalised hernial meshes. Eight different meshes with and without the loading of an antibiotic (ciprofloxacin $\mathrm{HCl}$ ) were designed using two different polymers, polypropylene (PP) and polyvinyl alcohol (PVA), having different pore size, shape and thread thickness. Printed meshes were evaluated for their mechanical, drug loading and release characteristics. Among the fabricated meshes, polypropylene has shown adequate printability characteristics where polyvinyl alcohol filaments showed comparatively easy handling during 3D printing. All the printed meshes showed satisfactory mechanical properties. However, the PVA meshes showed slightly faster release as compared to PP based meshes. Moreover, in-vivo testing in rabbit models was also performed for assessing biocompatibility and adhesiogenecity. Post-implantation observations in animal models revealed no signs of implant rejection and the extent of adhesions to the visceral tissue was mild to moderate. Animals implanted with ciprofloxacin $\mathrm{HCl}$ loaded meshes exhibited fewer fluctuations in body temperature, and they had faster-wound healing capacity. This work demonstrated for the first time that FDM is an effective and low-cost alternative for the manufacturing of tailored mesh for the management of hernia. The method has also been successfully employed for the preparation of drug loaded 3D mesh, which may be effectively used against post-surgical infections.

Keywords: 3D printing; fused deposition modeling; hernia mesh; ciprofloxacin $\mathrm{HCl}$; tensile strength; polypropylene; polyvinyl alcohol. 


\section{1- Introduction}

The hernia is a human body internal disorder in which an organ protrudes through a weak spot in the muscle or an immediate tissue wall ${ }^{[1]}$. There are several forms of hernia which can be located in different body parts. The most common hernias are inguinal, ventral, incisional, umbilical, femoral, epigastric and hiatal ${ }^{[2]}$. It is typically repaired by surgical intervention, and there is no established standard technique for this procedure ${ }^{[3,4]}$. Post-operative infection and recurrence are essential problems associated with their management/repair. The most common technique of hernia repair is the implantation of polymeric mesh at the defect site in order to support the damaged tissue until the herniated area can be sealed by new tissue growth. This procedure results in a lower recurrence rate and fewer complications than suture repair ${ }^{[5]}$.

The first mesh for hernia repair was made of polyethylene, and it was introduced by Usher et al. ${ }^{[6]}$. Nowadays, Polypropylene (PP) is the most commonly used polymer for the fabrication of non-absorbable hernial mesh, but the dilemma of chronic pain, post-operative infection and hernia recurrences persists ${ }^{[2,7]}$ and adaptations to this mesh are under investigation in order to enhance the clinical outcome ${ }^{[8,9]}$. The ideal mesh must permit the repair of the fundamental fascial defect by incorporating it into the surrounding body tissue while maintaining little adhesions and high tensile strength. It must not forbid; tissue growth, peritoneal regeneration and progress of the regular healing process ${ }^{[10]}$. An antibiotic-loaded hernial mesh that releases the drug at the surgery site may be an effective way of preventing postoperative infection ${ }^{[11}$, 12].

One conventional method for the fabrication of hernial mesh weaving polymer fibres. However, this is a time-consuming process that results in high costs. In the proposed study, a 3-D printing technique was used for printing hernial meshes to develop a customised mesh product with or without the loading of antibiotic, ciprofloxacin $\mathrm{HCl}$. 3D printing also referred 
as rapid prototyping (RP) or additive manufacturing (AM) which was initiated by Charles Hull in 1986. Currently, several 3D printing techniques are being employed, and these include stereo lithography (SLA), selective laser sintering (SLS), inject printing and fused deposition modeling $(\mathrm{FDM})^{[13]}$. However, among these, FDM is the most promising and widely used 3D printing technique for the development of customised and personalised dosage forms and implants ${ }^{[14]}$. In this technique, polymer filament is extruded after heating to its melting point through a nozzle; this nozzle moves above the base plate guided by the computer where the polymer solidifies and acquires the desired shape. The possibility to select a wide range of printing parameters such as the size of droplets, wall thickness, infill percentages and pattern types makes this technique ideal for the fabrication of a solid dosage form ${ }^{[15,16]}$. In the past few years, many types of drug delivery systems have been developed through FDM, and these include pharmaceutical implants, fast and extended drug release devices ${ }^{[17,18]}$.

This study is aimed at fabrication of on-demand, patient-specific surgical meshes through FDM; for improving the clinical outcome, enhancing technical capacity, reducing wastage and minimising the cost. Meshes with different pore sizes and shapes can be produced in a short time using several types of biocompatible polymeric materials. Moreover, antibiotic-loaded meshes can be printed in order to minimise post herneoplasty infections ${ }^{[19-21]}$. This study has made use of FDM for the production of a variety of surgical meshes with different specifications of pore size and shape, and it has also employed two different types of polymers (PP and PVA) with and without the loaded drug. Ciprofloxacin $\mathrm{HCl}$ was chosen as a model antibacterial agent used for complicated abdominal or urinary tract infections ${ }^{[22,23]}$. Also, this antibiotic has shown the potential to penetrate and destroy bacterial biofilms ${ }^{[24]}$. Therefore, it can be hypothesised that the antibiotic-loaded hernia meshes may prove useful for the prophylaxis of post-operative infection. To the best of our knowledge, this is the first 
comprehensive study of developing plain and drug loaded hernial meshes using FDM. Moreover, the printed meshes were characterised for mechanical, thermal and drug release properties. Also, an in-vivo study (in the rabbit model) was also carried out for studying the biocompatibility and adhesiogenecity of newly developed meshes.

\section{Materials and methods}

\subsection{Materials}

\subsubsection{Filaments}

Polypropylene, (which has a melting point of $170{ }^{\circ} \mathrm{C}$ and printing temperature of $190{ }^{\circ} \mathrm{C}$ ) and poly vinyl alcohol (which has a melting point of $190{ }^{\circ} \mathrm{C}$ and a printing temperature of $200^{\circ} \mathrm{C}$ ) and filaments purchased from Maker Bot, USA were used as already extruded filaments with a diameter of $1.75 \mathrm{~mm}$ and they were compatible with a maker boat $2 \mathrm{x}$ replicator for fused deposition modeling.

\subsubsection{Drug}

Ciprofloxacin $\mathrm{HCl}$ (which has a melting point $314{ }^{\circ} \mathrm{C}$ ), is a broad spectrum synthetic antibiotic and it was acquired as a gift from Harmann Pharmaceuticals, Lahore, Pakistan. The identification of the drug was determined by FTIR (Fourier-transform infrared spectroscopy). The stated aqueous solubility of ciprofloxacin hydrochloride according to the manufacturer's literature was $35 \mathrm{mg} / \mathrm{ml}$.

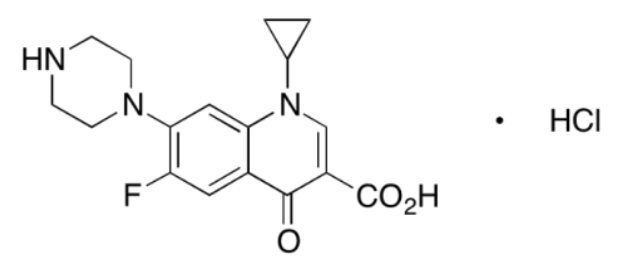

Figure 1. Chemical structure of ciprofloxacin $\mathrm{HCl}$ 


\subsubsection{Reference mesh}

Commercially available prolene mesh by Ethicon ${ }^{\circledR}$ was used as reference for in-vitro and invivo experiments. It is a non-absorbable, woven mesh with a pore size of $0.2 \mathrm{~mm} \times 0.1 \mathrm{~mm}$ and is most commonly used in hernioplasty ${ }^{[8]}$.

\subsection{Methods}

\subsubsection{Solubility of the drug in ethanol and water}

The solubility of the drug in ethanol and water was measured separately using the shake flask method ${ }^{[25]}$. The excess amount of ciprofloxacin $\mathrm{HCl}(\sim 1 \mathrm{~g}$ and $5 \mathrm{~g}$ for ethanol and water respectively) was added to a conical flask containing $50 \mathrm{ml}$ of solvent. The mixture was maintained at $37 \pm 1{ }^{\circ} \mathrm{C}$ and was shaken continuously for 24 hours. It was allowed to stand for another 16 hours. The supernatant was filtered using a membrane filter (pore size $0.45 \mu \mathrm{m}$ ) before sampling. The aliquots of filtrate $(1 \mathrm{ml})$ were removed and diluted with water. The concentration was determined by a calibrated UV spectrophotometer (UV-2550 by Shimadzu) at $\lambda_{\max }$ of $273 \mathrm{~nm}$ and all the measurements were carried out in triplicate.

\subsubsection{Drug loading}

For PP filament drug loading, the ciprofloxacin $\mathrm{HCl}$ solution in water was prepared by dissolving $5 \mathrm{~g}$ of the drug in $250 \mathrm{ml}$ of water. A piece of PP filament equal to $15 \mathrm{~g}$ was immersed in this solution. It was then placed over a hot plate magnetic stirrer at a speed of $1000 \mathrm{rpm}$ and maintained at $40{ }^{\circ} \mathrm{C}$ for 24 hours. Afterwards, the filament was taken out, and it was air dried and kept protected from light for further processing.

For PVA, the saturated dispersion of ciprofloxacin $\mathrm{HCl}$ was prepared by mixing $\sim 5 \mathrm{~g}$ of the powdered drug with $250 \mathrm{ml}$ of absolute ethanol ( 99.7\%). A piece of PVA filament $(15 \mathrm{~g})$ was immersed in the above dispersion and placed in a sonicator for 12 hours. The filament was then 
kept in a tray and air dried to a constant weight and finally stored in desiccators in a dark place for further analysis.

\subsubsection{Drug assay}

A series of standard solutions with concentration ranges from $0.1 \%$ to $10 \%$ were prepared by dissolving the required amount of ciprofloxacin $\mathrm{HCl}$ in $0.1 \mathrm{~N} \mathrm{HCl}$. Dilutions were then made with $0.1 \mathrm{~N} \mathrm{HCl}$ to the desired concentration. The absorbance was determined using a calibrated spectrophotometric method with a $\mathrm{R}^{2}$ value of 0.998 at $\lambda_{\max }$ of $273 \mathrm{~nm}$. For the sample analysis, $500 \mathrm{mg}$ of the drug loaded filament was soaked in $60 \mathrm{ml}$ of $0.1 \mathrm{~N} \mathrm{HCL}$ in a $100 \mathrm{ml}$ volumetric flask and it was sonicated for 12 hours. The solution was filtered and the volume was made up to the mark with $0.1 \mathrm{~N} \mathrm{HCl}$ solution. $1 \mathrm{ml}$ of this solution was then diluted to $100 \mathrm{ml}$ with $0.1 \mathrm{~N}$ $\mathrm{HCl}$ for drug analysis by the above described UV method. All the experiments were carried out in triplicate.

\subsubsection{Differential scanning calorimetry (DSC)}

The differential scanning calorimetry Q 2000 by TA instruments was used to perform DSC measurements of the polymers in both drug loaded and unloaded states. Aluminum pans with a capacity of 8-10 mg were used. The heating rate was kept at $10{ }^{\circ} \mathrm{C}$ per minute. All the experiments were performed using nitrogen as purge gas which was flowing at a rate of $50 \mathrm{ml}$ per minute. The heating range was kept between $20-350{ }^{\circ} \mathrm{C}$. The data analysis was performed using the TA instruments' universal analysis 2000 software.

\subsubsection{Design of mesh}

The template that was used to print the mesh was designed using solid works ${ }^{[26]}$ with different specifications of pore size, shape and thread diameter as shown in Table 1. The developed designs were saved .stl format which is compatible printing formate for Maker Boat printers. 
Table 1. Design specification used for printing of mesh

\begin{tabular}{|l|c|c|c|c|}
\hline Design & Pore size & Thread width & pore shape & Number of pores/100cm $^{2}$ \\
\hline D 1 & $3 \mathrm{~mm}$ & $0.8 \mathrm{~mm}$ & Diamond & 676 \\
\hline D 2 & $3 \mathrm{~mm}$ & $0.8 \mathrm{~mm}$ & Square & 676 \\
\hline D 3 & $2 \mathrm{~mm}$ & $0.8 \mathrm{~mm}$ & Diamond & 1225 \\
\hline D 4 & $2 \mathrm{~mm}$ & $0.8 \mathrm{~mm}$ & Square & 1225 \\
\hline D 5 & $3 \mathrm{~mm}$ & $1.2 \mathrm{~mm}$ & Diamond & 529 \\
\hline D 6 & $3 \mathrm{~mm}$ & $1.2 \mathrm{~mm}$ & Square & 529 \\
\hline D 7 & $2 \mathrm{~mm}$ & $1.2 \mathrm{~mm}$ & Diamond & 900 \\
\hline D 8 & $2 \mathrm{~mm}$ & $1.2 \mathrm{~mm}$ & Square & 900 \\
\hline
\end{tabular}

\subsubsection{Printing of mesh}

By incorporating four types of filaments (two drug loaded and two plain filaments of PP and PVA) and eight designs (as described in Table 1), 32 theoretical formulations with specifications as described in Table 1 were expected. These formulations were fabricated using a commercial fused-deposition modeling 3D printer and a MakerBot Replicator 2 Desktop 3D printer (MakerBot Inc, USA). The selected size for the mesh was X = $100 \mathrm{~mm}$ (major length), $\mathrm{Y}=100 \mathrm{~mm}$ (minor length) and $\mathrm{Z}=0.8 \mathrm{~mm}$ (height). The printer settings were adjusted to produce the best meshes for all the filaments. The standard resolution with the raft option was deactivated and the extrusion temperatures were $190{ }^{\circ} \mathrm{C}$ and $200{ }^{\circ} \mathrm{C}$ for PP and PVA, respectively. The speed while extruding and traveling was set at $90 \mathrm{~mm} / \mathrm{s}$ and150 mm/s, respectively. The infill percentage was kept constant at $90 \%$ for all the printed meshes.

\subsubsection{Mechanical properties of filament and printed mesh}

The mechanical properties such as strain at break and the percent elongation of printed meshes were determined by using the universal material testing machine (Lloyd instruments, UK). All the printed and reference mesh samples were cut to same width of $15 \mathrm{~mm}$ and length was kept at $50 \mathrm{~mm}$ greater than the initial grip separation used for each type of mesh that was $50 \mathrm{~mm}$ for 
PP mesh and $125 \mathrm{~mm}$ for PVA mesh. The initial strain rate was $0.1 \mathrm{~mm} / \mathrm{min}$ for PVA and 10 $\mathrm{mm} / \mathrm{min}$ for PP mesh, whereas the rate of grip separation was adjusted at $12.5 \mathrm{~mm} / \mathrm{min}$ for PVA and $500 \mathrm{~mm} / \mathrm{min}$ for PP mesh. The thickness of each specimen was $0.8 \mathrm{~mm}$ and it was measured to be uniform to $5 \%$ of the thickness over the entire length. Test specimens were conditioned at $23 \pm 2{ }^{\circ} \mathrm{C}$ and at a relative humidity of $50 \pm 5 \%$ and then analysed under the same conditions of temperature and humidity. A load range of $10-160 \mathrm{~N}$ for PP and $0.1-100$ $\mathrm{N}$ for PVA was selected after a few trials so that breakage happened within its upper two thirds. The load versus extension was recorded for all samples both longitudinally and transversally and all the readings were taken in triplicate. The tensile strength and percent elongation of printed mesh was computed.

\subsubsection{Dissolution testing}

The meshes prepared from the drug loaded filaments were subjected to release studies by using the USP type V (Paddle over disc) apparatus. The phosphate buffer ( $\mathrm{pH} 7.4$ ) was maintained at $37 \pm 01{ }^{\circ} \mathrm{C}$ and it was used as a dissolution medium. A small piece of drug loaded mesh (2.5 $\mathrm{cm} \times 2.5 \mathrm{~cm}$ ) was attached to the glass disk and placed at the bottom of the dissolution cylinder. The speed of paddle was adjusted to $25 \mathrm{rpm}$. The sampling was done at one hour intervals for PP mesh and at 30 minutes intervals for PVA mesh. The dissolution medium was replenished with the same medium at each interval to maintain the sink conditions. The drug concentration was calculated using the UV spectrophotometric method. This process continued for 12 hours and six hours for PP and PVA mesh respectively.

\subsubsection{In-vivo testing for biocompatibility, adhesiogenecity and histologic characterization}

The in vivo study was conducted using the rabbit model as described by ${ }^{[27]}$. The testing was aimed at observing if there was any presence of immunologic response to the test samples and 
the tests were meant to evaluate their adhesiogenecity. The histologic examination of tissue growth was also performed to see if the wound would be cured. The protocols used for this study were approved by the Animal Ethical Committee, the University College of Pharmacy, University of the Punjab, Lahore, Pakistan (Ref no. AEC/PUCP/1072A). A group of healthy male rabbits (20 in total) weighing approximately $1 \mathrm{~kg}$ each were used. Commercially available hernia mesh (Prolene $\left.{ }^{\circledR}\right)$ was used as reference material to compare the results. Animals were kept in controlled temperature and light conditions and they were fed a fibre rich diet.

\subsubsection{Test materials}

One mesh type of each material (PVA, PP, drug loaded PVA and drug loaded PP) were selected for in vivo testing. Meshes printed with design \# 6 (pore size $3 \mathrm{~mm}$ and filament width $1.2 \mathrm{~mm}$ ) showed better printability and mechanical properties, therefore, they were selected for these studies. These were mesh 6 for PP, mesh 14 for drug loaded PP, mesh 22 for PVA and mesh 30 for drug loaded PVA. Commercially available prolene mesh was used as a reference. Size $1.5 \mathrm{~cm} \times 1.5 \mathrm{~cm}$ specimens were cut from each type of mesh in order for them to be tested and sterilised by standard procedure.

\subsubsection{Implant surgery}

Each rabbit was weighed before surgery and its abdomen was shaved and cleaned. For anesthetising, $30 \mathrm{mg}$ per $\mathrm{kg}$ of ketamine and $5 \mathrm{mg} / \mathrm{kg}$ of xylazine were injected intramuscularly in to each rabbit. The pictorial representation of the implant surgery is shown in Figure 2 below. A $1 \mathrm{~cm}$ incision was made along the midline of the abdomen after the induction of the anesthesia. Approximately, a $1 \mathrm{~cm}$ wide defect was made in the abdominal fascia and the abdominal viscera was exposed. The mesh specimen was placed below the defect and stitched to the muscle tissue using prolene 3-0 round body thread. Silk 3-0 thread was used to stitch the subcutaneous tissue. After recovering from anesthesia, all animals were kept under supervision 
and they were continuously monitored for temperature, pulse, wound healing, eating and defecating habits.
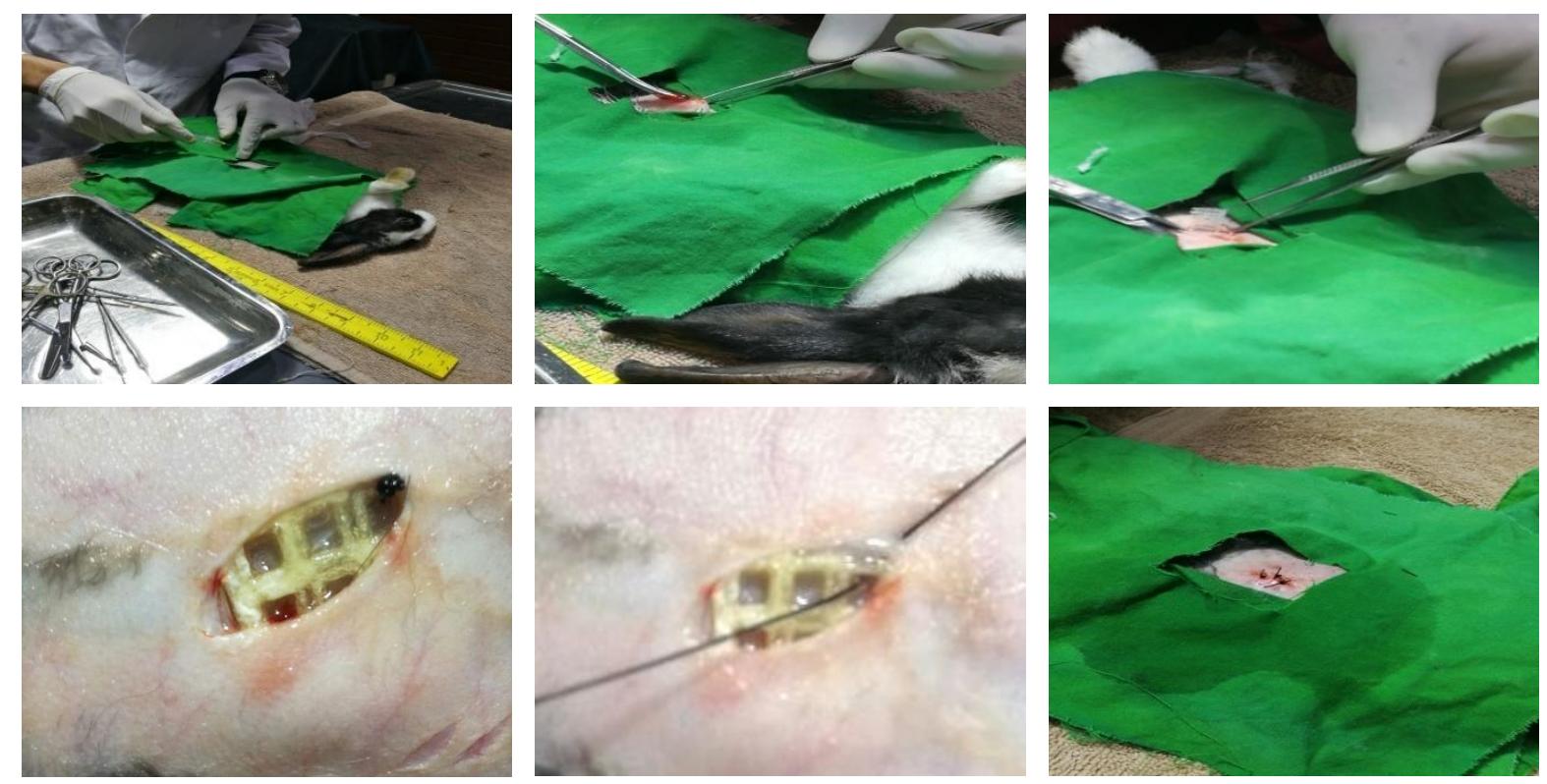

Figure.2. Steps of implant surgery

\subsubsection{Wound healing and clinical evaluation}

Some ointment was applied throughout the wound healing process in order to avoid wound dryness. The external stitches were removed after seven days. The healing process was carefully observed for inflammation at the site of surgery and for any change in food intake and activity. The body temperature was checked twice every day at feeding times.

\subsubsection{Explant surgery}

Rabbits were euthanised by using $25 \mathrm{mg}$ Ketamine per kg, 2.5mg Acepromazine per kg and $5 \mathrm{mg}$ Xylazine per $\mathrm{kg}$, this was followed by a $3 \mathrm{ml}$ Euthasol cardiac injection. Euthasol is a combination of Phenytoin Sodium and Phenobarbital Sodium. Thereafter, abdominal incisions were made, implants were found and the extent of adhesions and resistance to separation 
(tenacity) was noted and evaluated against the scale given in Table.2. After separation, implants were kept in 10 percent formalin solution and studied for histology.

Table 2. Adhesion scoring scale used in this study.

\begin{tabular}{|l|l|l|}
\hline \multirow{2}{*}{$\begin{array}{l}\text { Extent (\% area of } \\
\text { implant } \\
\text { involved) }\end{array}$} & Grade & Trait \\
\cline { 2 - 3 } & 1 & Adhesions are absent \\
\cline { 2 - 3 } & 2 & Up to $25 \%$ adhesions covering the implant \\
\cline { 2 - 3 } & 3 & Up to50\%adhesions covering the implant \\
\cline { 2 - 3 } & 4 & Up to75\%adhesions covering the implant \\
\hline $\begin{array}{l}\text { Resistance to } \\
\text { (tenacity) }\end{array}$ & 0 & Up to100\%adhesions covering the implant \\
\cline { 2 - 3 } & 1 & Mdhesions are absent \\
\cline { 2 - 3 } & 2 & Moderate \\
\cline { 2 - 3 } & 3 & Sharp dissection required. \\
\cline { 2 - 3 } & 4 &
\end{tabular}

\section{Results and discussion}

\subsection{Drug loading and DSC studies}

Drug loading in PP filaments was achieved through an aqueous solution, owing to the high solubility of ciprofloxacin $\mathrm{HCl}$ in water. For PVA filament, it was achieved by using ethanolic solution rather than the aqueous solution. The latter would result in an undesirable swelling of PVA filament and possible dissolution. The drug loading into both filaments followed the same procedure as used for hydrogels ${ }^{[28]}$. Polymer filament was soaked in drug dispersion followed by drying at controlled temperature and humidity conditions. The process of dipping the polymer filament in the drug solution is based on the supposition that the drug and the polymer do not interact chemically and the drug loading takes place through passive diffusion or adsorption. The drug is loaded to the polymer upon drying which guarantees that the filament diameter does not change so it is printed without any trouble. The adopted method was robust and needed little development other than the selection of a suitable solvent. The ultimate drug 
content of the filaments was $3 \pm 1 \%$ w/w for PP and $5 \pm 1 \%$ w/w for PVA. In contrast, to other studies where $<1 \% \mathrm{w} / \mathrm{w}$ drug loading was achieved, these results show enhanced drug loading which can be attributed to the nature of the drug and the selection of an appropriate solvent. Both polymers and their drug combinations were subjected to thermal analysis before printing (Figure 3). This analysis is important in accessing the suitability of the use of the 3D process for selected drug and filaments. The DSC curve of PP filament (Figure 3A) shows a melting event at $\sim 162{ }^{\circ} \mathrm{C}$ and there is no evidence for its degradation up to $250{ }^{\circ} \mathrm{C}$ thereby representing its high thermal stability. The TGA curve shows a small weight loss at melting point and severe weight loss after $250{ }^{\circ} \mathrm{C}$, thereby representing the degradation of the polymer. The thermal behaviour of PVA filament is shown in (Figure 3B), which is notably different from the PP. It shows a shallow endotherm of $\sim 190{ }^{\circ} \mathrm{C}$ representing its melting (Figure 3B). The TGA curve shows a small mass loss prior to this melting event ascribed to the loss of water. At temperatures higher than $280^{\circ} \mathrm{C}$, the PVA is seen to undergo thermal degradation as evidenced by the severe weight loss. These findings are consistent with the previously published results ${ }^{[18]}$. Ciprofloxacin $\mathrm{HCl}$ is seen to melt at $\sim 318{ }^{\circ} \mathrm{C}$ with significant mass loss (Figure 3C). It is important to note that the melting range of ciprofloxacin is observed to be the same when its combinations with PVA and PP are analyzed and no significant noise is seen in the DSC data which suggests that there is a lack of interaction between the drug and polymer (Figures 3DE). It is pertinent to note that the printing temperature of PVA and PP is $200{ }^{\circ} \mathrm{C}$ and $190{ }^{\circ} \mathrm{C}$ respectively, which is much lower than the degradation of PVA, PP and the drug, thereby showing that there is no risk of degradation of the drug during the printing process. 

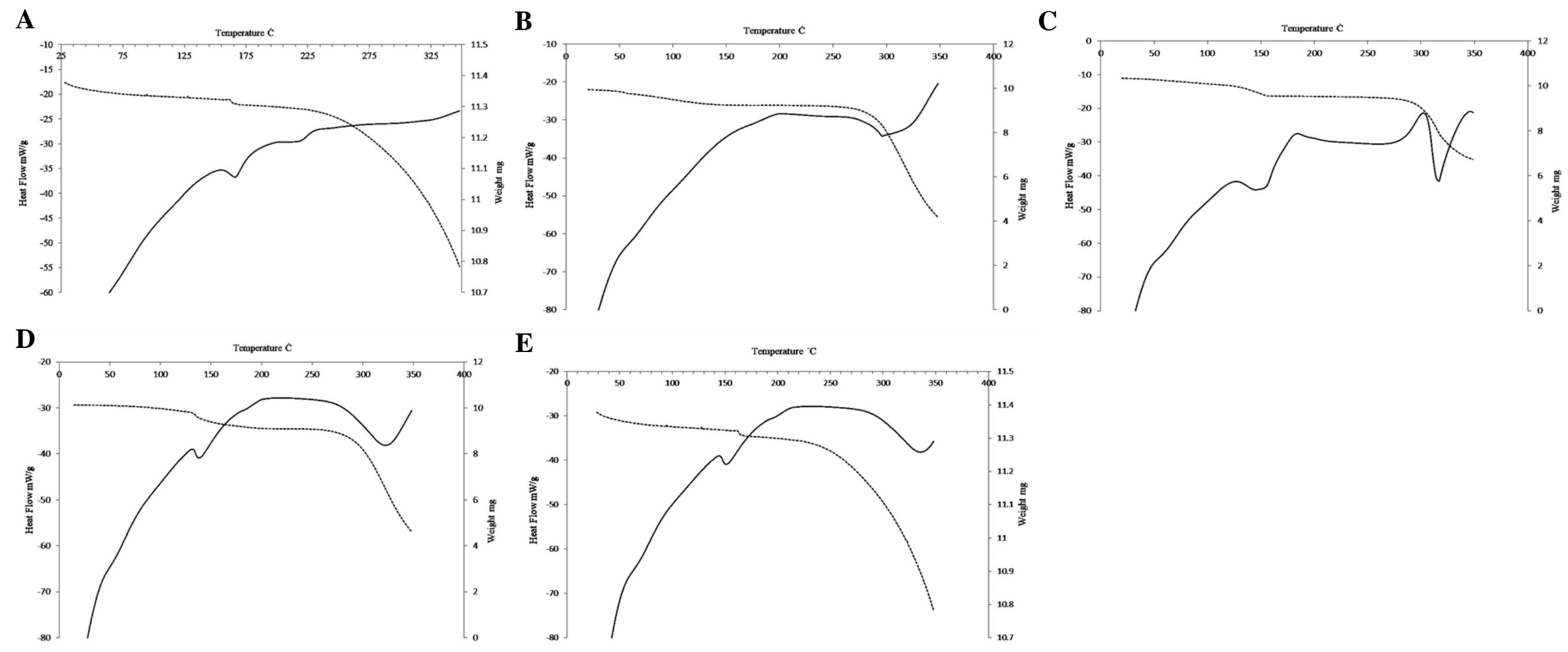

Fig. 3. TGA and DSC plots for (A) Plain PP filament (B) Plain PVA filament (C) Ciprofloxacin HCl (D) Drug loaded PP (E) Drug loaded PVA, (for interpretation, the dotted line shows the TGA curve and the solid line shows the DSC curve). 


\subsection{Fabrication of 3D printed hernia meshes}

The fabrication of polymeric meshes incorporating four types of filaments (two ciprofloxacin $\mathrm{HCl}$ loaded and two plain filaments of PP and PVA) was achieved using a commercially fuseddeposition modeling 3D printer known as the MakerBot Replicator 2 Desktop 3D printer (MakerBot Inc, USA). A design template is an important parameter for the printing and strength of hernial mesh. In total, eight designs with different pore shapes, pore sizes and thread thickness were tested for printability and later for tensile strength. Theoretically, 32 formulations with specifications described in Table 1 were expected but practically only 20 formulations (16 for PP and 4 for PVA, as in Table 3) could be printed. Figure 4 shows pictures of printed meshes from both filaments.

There have been certain limitations to the above mentioned technique for the fabrication of hernia meshes. Splitting of threads was observed in meshes printed with larger pore size. Because of hygroscopic nature of PVA, very fine printing was not possible, the designs with a minimum pore size of $3 \mathrm{~mm}$ and thread diameter $1.2 \mathrm{~mm}$ could be printed. Whereas for PP, all designs were successful and showed good printing behavior in both drug loaded and plain forms. 


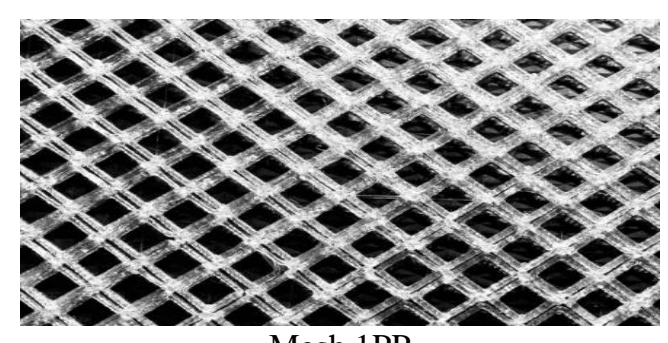

Mesh 1PP

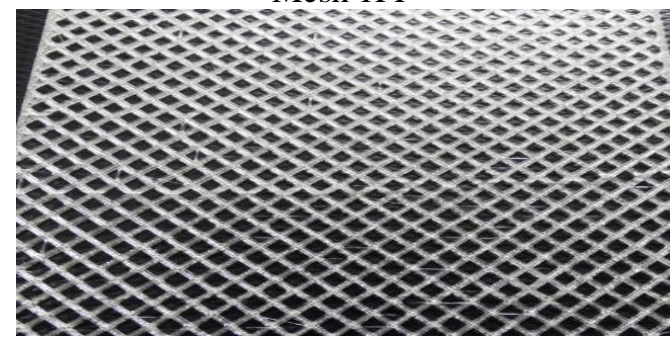

Mesh 3PP

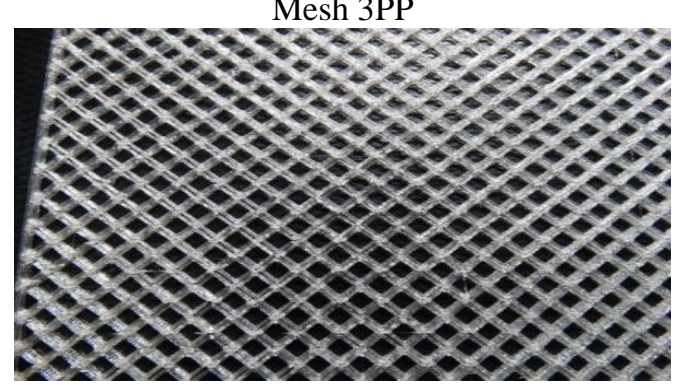

Mesh 5PP

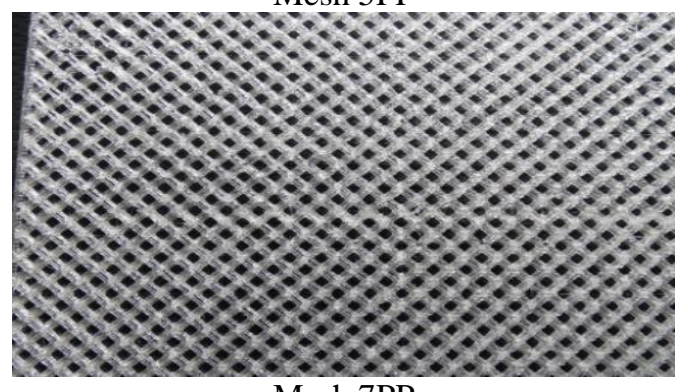

Mesh 7PP

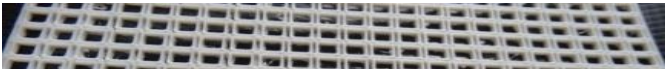

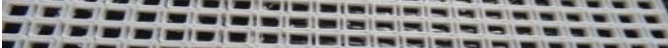

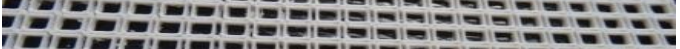

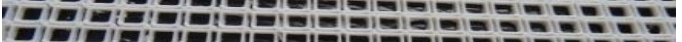

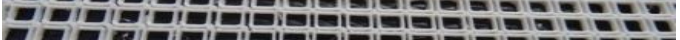

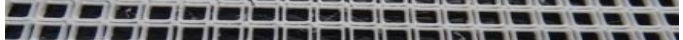

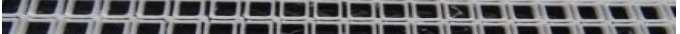

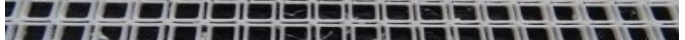

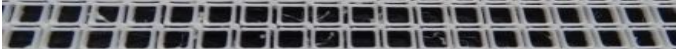

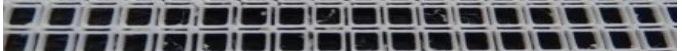

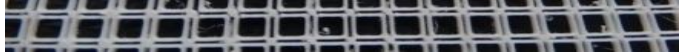
Mesh 22PVA

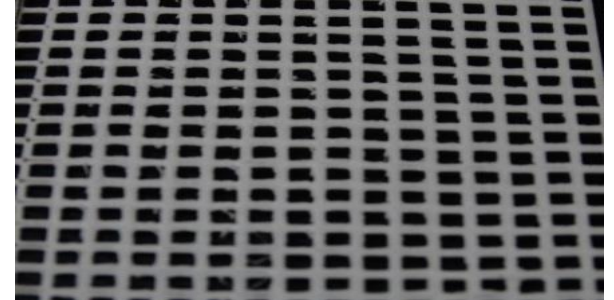

Mesh 2PP

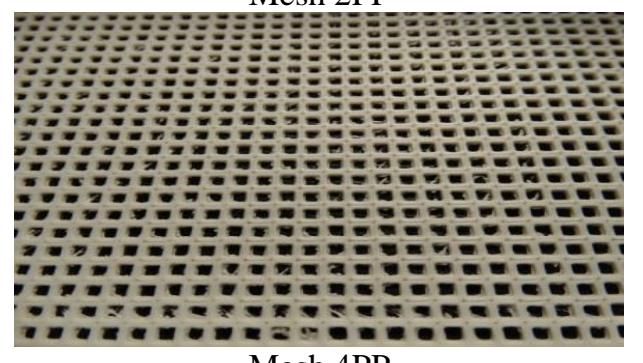

Mesh 4PP

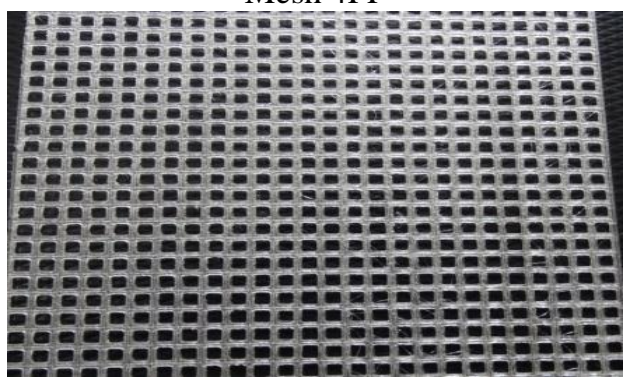
Mesh 6PP

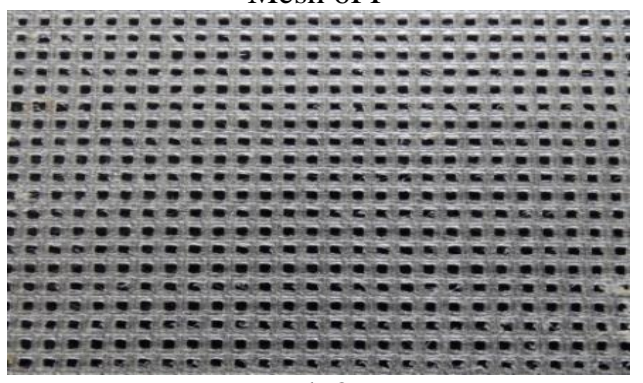
Mesh 8PP

- - - - - - - - - - - - -

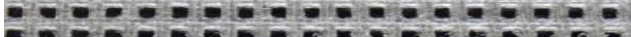

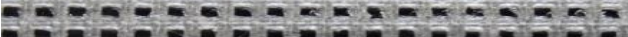

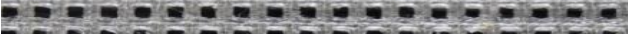

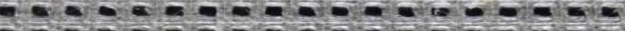

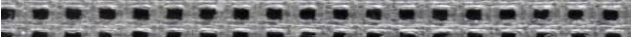

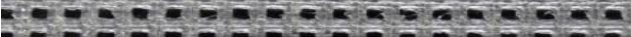

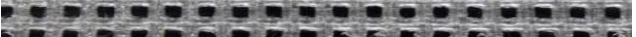

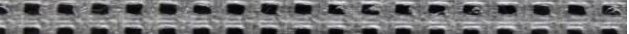

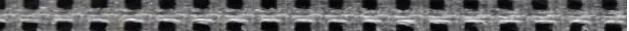

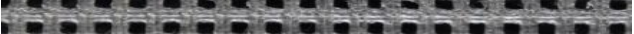

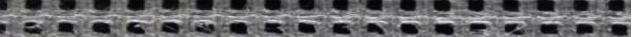

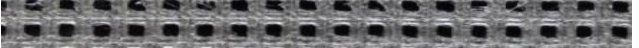
Mesh 24PVA

Figure 4. Pictures of printed meshes (Taken by DSLR camera).

(for clarity only one mesh from each successful design template is shown here) 


\subsection{Mechanical properties of filament and printed mesh}

Mechanical properties of surgical meshes are normally determined by measuring tensile strength and percent elongation. The tensile testing of unloaded filaments and meshes (Table

3) revealed that PVA filament was much stronger and stiffer as compared to PP filament. It is important to note that the tensile strength for 3D printed PVA meshes was 2 to 3 times lower than the filament itself, whereas the tensile strength for 3D printed PP meshes was comparable to that of the filament. Mesh designs with a larger thread width and smaller pore size were stronger than the others. Drug loading had no significant effect on the tensile properties of PP meshes but the tensile strength of PVA meshes was reduced in the drug loaded state. Previous studies suggest that for hernia repair, a tensile strength of $16 \mathrm{~N} / \mathrm{cm}^{2}$ is sufficient to augment the abdominal wall, however, for bridging of large defects, a tensile strength of $32 \mathrm{~N} / \mathrm{cm}^{2}$ may be necessary [29]. The results of this study have shown that the tensile strength of 3D printed PP meshes ranges from $25 \mathrm{~N} / \mathrm{cm}^{2}$ to $53 \mathrm{~N} / \mathrm{cm}^{2}$ which is greater than the suggested strength, however, the tensile strength of PVA meshes ranges from $17 \mathrm{~N} / \mathrm{cm}^{2}$ to $30 \mathrm{~N} / \mathrm{cm}^{2}$ which is within the acceptable range. The tensile strength of $3 \mathrm{D}$ printed mesh increases with the increase in the thread diameter and a decrease in the pore size. The meshes that were printed with the D7 and D8 designs (pore size $2 \mathrm{~mm}$, thread diameter $1.2 \mathrm{~mm}$ ) showed the greatest tensile strength. The percent elongation for PP meshes ranged from 300-450\% whereas for PVA meshes it ranged from $10-20 \%$. The pore shape and the direction of sample testing (longitudinal or transverse) had no significant effect on the tensile properties of the 3D printed meshes in contrast to the reference mesh which showed a difference in tensile properties in transverse and longitudinal directions. The weight of the PP meshes ranged from $2.9 \mathrm{~g} / \mathrm{cm}^{2}$ to $3.5 \mathrm{~g} / \mathrm{cm}^{2}$ which were comparable to the reference mesh $\left(3.1 \mathrm{~g} / \mathrm{cm}^{2}\right)$. PVA meshes were expectedly heavier than PP meshes owing to the higher specific gravity of PVA. The mesh weight increased by increasing the thread width and reducing the pore size (design no. D7, D8), while the meshes with a 
smaller thread width and larger pore size (D1, D2) were the lightest. Although the reference mesh has smaller pore size than the printed ones, our formulation has shown desirable characteristics for hernia repair.

Table.3. Tensile properties of printed meshes.

\begin{tabular}{|c|c|c|c|c|c|c|}
\hline \multirow[b]{2}{*}{$\begin{array}{l}\text { Mesh } \\
\text { code }\end{array}$} & \multirow[b]{2}{*}{ Design } & \multirow[b]{2}{*}{$\begin{array}{l}\text { Drug } \\
\text { loading }\end{array}$} & \multicolumn{2}{|c|}{ Longitudinal } & \multicolumn{2}{|c|}{ Transverse } \\
\hline & & & $\begin{array}{c}\text { Tensile } \\
\text { strength }\left(\mathrm{N} / \mathrm{cm}^{2}\right)\end{array}$ & $\begin{array}{c}\text { Percent } \\
\text { elongation } \\
(\%)\end{array}$ & $\begin{array}{c}\text { Tensile } \\
\text { strength }\left(\mathrm{N} / \mathrm{cm}^{2}\right)\end{array}$ & $\begin{array}{c}\text { Percent } \\
\text { elongation } \\
(\%)\end{array}$ \\
\hline \multicolumn{2}{|c|}{ Reference Mesh } & - & 79 & 390 & 64 & 420 \\
\hline $1 \mathrm{PP}$ & D1 & - & 25 & 385 & 24.3 & 390 \\
\hline $2 \mathrm{PP}$ & $\mathrm{D} 2$ & - & 27.5 & 375 & 26.9 & 370 \\
\hline $3 \mathrm{PP}$ & D3 & - & 33.3 & 365 & 32.3 & 364 \\
\hline $4 \mathrm{PP}$ & D4 & - & 35.8 & 370 & 34.4 & 370 \\
\hline $5 \mathrm{PP}$ & D5 & - & 34.1 & 360 & 33.9 & 365 \\
\hline $6 \mathrm{PP}$ & D6 & - & 35.1 & 380 & 34.3 & 375 \\
\hline $7 \mathrm{PP}$ & D7 & - & 53.3 & 395 & 52.2 & 390 \\
\hline $8 \mathrm{PP}$ & D8 & - & 54.1 & 388 & 53.1 & 392 \\
\hline $9 \mathrm{PP}$ & D1 & + & 24.3 & 370 & 23.2 & 375 \\
\hline $10 \mathrm{PP}$ & D2 & + & 26.9 & 375 & 25.6 & 368 \\
\hline $11 \mathrm{PP}$ & D3 & + & 32.3 & 385 & 31.5 & 390 \\
\hline $12 \mathrm{PP}$ & D4 & + & 34.4 & 384 & 33.9 & 388 \\
\hline $13 \mathrm{PP}$ & D5 & + & 33.9 & 388 & 31.2 & 386 \\
\hline $14 \mathrm{PP}$ & D6 & + & 34.3 & 390 & 33.4 & 395 \\
\hline $15 \mathrm{PP}$ & D7 & + & 52.2 & 395 & 51.3 & 400 \\
\hline $16 \mathrm{PP}$ & D8 & + & 53.1 & 410 & 53 & 405 \\
\hline 22 PVA & D6 & - & 27.5 & 26.5 & 26.5 & 10.6 \\
\hline 24 PVA & D8 & + & 29.1 & 28.2 & 28.2 & 11.2 \\
\hline $30 \mathrm{PVA}$ & D6 & - & 19.8 & 17.5 & 17.5 & 16 \\
\hline 32 PVA & D8 & + & 20 & 19.4 & 19.4 & 19 \\
\hline
\end{tabular}




\subsection{Drug release studies}

The dissolution profile of the drug from the meshes prepared from the drug loaded filaments (mesh no. 16 PP and 32 PVA) is shown in Figure 5. PP and PVA meshes release $~ 60 \%$ and $\sim 70 \%$ of the drug within the first hour respectively. Plateau was achieved within 6 to 7 hours for both filaments.

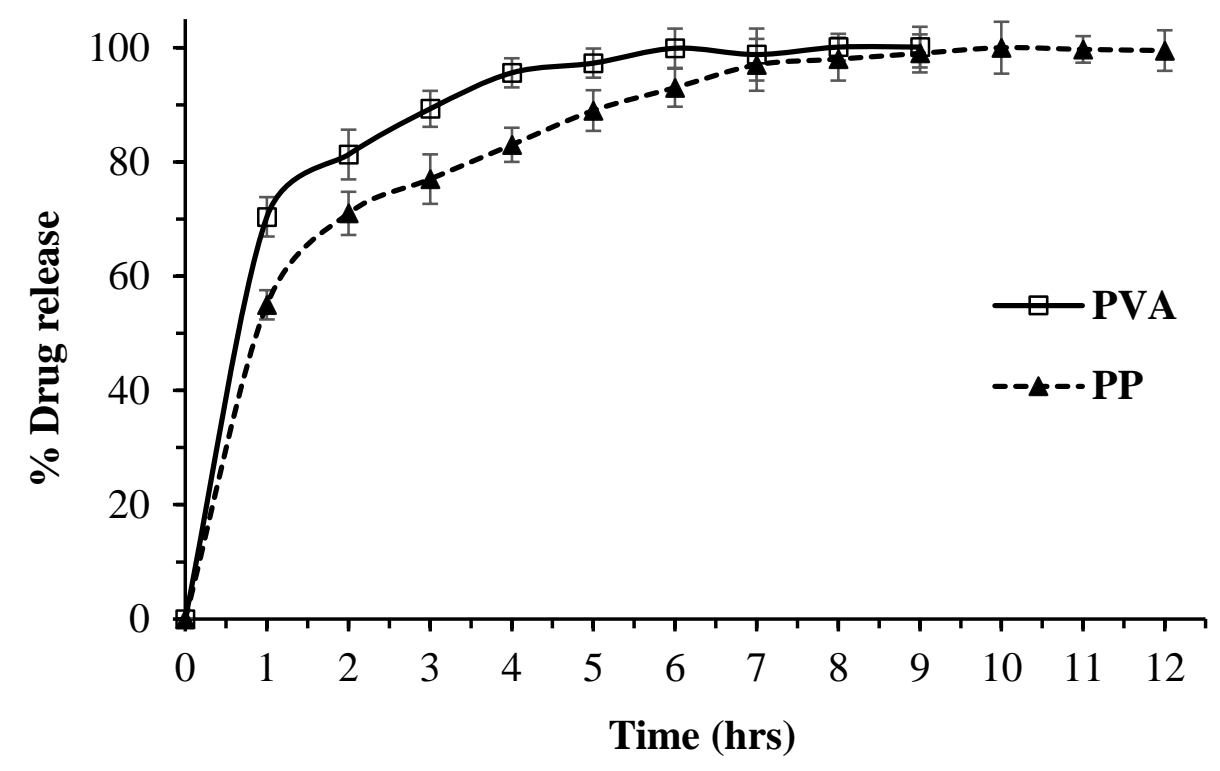

Figure 5. Drug release profiles of PP and PVA meshes.

\subsection{In vivo evaluation}

Although a clinical study is the best means to evaluate the outcome of new mesh formulation, preclinical animal models are necessary for assessing biocompatibility and strength [30, 31]. A brief review of past research showed reports with rabbits, guinea-pigs, rats, pigs and dogs as models for evaluation of hernia meshes. Almost all the studies with a few exceptions were on 
wound healing as well as scar formation and they were sourced on the promotion of an acute full-depth defect in the abdominal wall that was patched up by a mesh and/or a surgical procedure [32-35]. Previous research suggests that a full-depth defect in a rabbit model was practicable and dependable to support an incisional hernia that could reproduce an appropriate pathophysiological state and could be used as a standard model to enhance the assessment of approaches to hernia repair [27].

The key purpose of this animal study was to observe immunologic response and reveal the adhesiogenecity properties of mesh formulations. The surgical procedures, anesthesia and revival were without any adverse incident in all animals and all stayed alive to their preset sacrifice date and they were evaluated. No change was observed in the drinking, eating, defecating or urinating habits of any rabbit in the period of study and all of them either gained or maintained weight throughout the experiment. No undesirable events, bulging or hernia formations were observed. Table 4 summaries all post-operative clinical observations for the study period. Animals implanted with ciprofloxacin $\mathrm{HCl}$ loaded meshes exhibited less fluctuations in body temperature and were faster in wound healing. All implanted meshes were integrated into the nearby tissues and seemed continuous with the adjacent body wall. The outer skin and underneath tissue were loosely attached to the mesh implants and no adhesions involving visceral tissue were seen. Comparatively, reference mesh had the largest extent and tenacity (resistance to separation) of adhesions. No visceral adhesions were observed in case of PVA meshes in the six week time period. 
Table 4. Post implantation observations in rabbit models.

\begin{tabular}{|c|c|c|c|c|c|c|c|c|}
\hline & \multirow[b]{2}{*}{ Model } & \multicolumn{2}{|c|}{ Temperature Fluctuation } & \multirow{2}{*}{$\begin{array}{l}\text { Wound } \\
\text { Healing }\end{array}$} & \multirow[b]{2}{*}{ Inflammation } & \multirow{2}{*}{$\begin{array}{c}\text { Weight } \\
\text { Variation }\end{array}$} & \multicolumn{2}{|c|}{ Adhesions* } \\
\hline & & $\begin{array}{l}\text { Minimum } \\
\text { (F) }\end{array}$ & $\begin{array}{c}\text { Maximum } \\
\text { (F) }\end{array}$ & & & & Extent & Tenacity \\
\hline \multirow{4}{*}{ 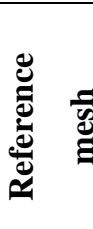 } & $\mathrm{R} 1$ & 101.1 & 103 & Delayed & Moderate & Maintained & 1 & 2 \\
\hline & $\mathrm{R} 2$ & 100.4 & 103.2 & Delayed & Moderate & Maintained & 1 & 2 \\
\hline & R3 & 100.3 & 102.5 & Delayed & Moderate & Gained & 1 & 2 \\
\hline & R4 & 99.5 & 101.7 & Normal & Mild & Gained & 1 & 1 \\
\hline \multirow{4}{*}{$\hat{z}$} & R5 & 100 & 102.6 & Normal & Mild & Maintained & 1 & 1 \\
\hline & R6 & 100.4 & 103.2 & Delayed & Moderate & Maintained & 1 & 1 \\
\hline & R7 & 101.7 & 103.6 & Delayed & Moderate & Maintained & 1 & 1 \\
\hline & R8 & 100.2 & 102.4 & Normal & Mild & Gained & 1 & 1 \\
\hline \multirow{4}{*}{ 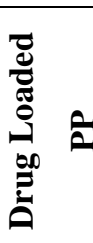 } & R9 & 98.8 & 101.5 & Normal & Mild & Maintained & 1 & 1 \\
\hline & $\mathrm{R} 10$ & 99 & 101.9 & Normal & Mild & Gained & 1 & 1 \\
\hline & R11 & 98.6 & 100.4 & Fast & Absent & Gained & 1 & 1 \\
\hline & R12 & 99.4 & 102.1 & Normal & Mild & Maintained & 1 & 1 \\
\hline \multirow{4}{*}{ 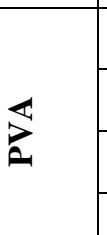 } & R13 & 98.8 & 102.8 & Normal & Mild & Maintained & 0 & 0 \\
\hline & R14 & 99 & 103.5 & Normal & Mild & Maintained & 0 & 0 \\
\hline & R15 & 99.1 & 102.6 & Normal & Mild & Gained & 0 & 0 \\
\hline & R16 & 98.6 & 102.1 & Normal & Absent & Maintained & 0 & 0 \\
\hline \multirow{4}{*}{ 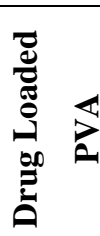 } & R17 & 98.3 & 99.4 & Fast & Absent & Gained & 0 & 0 \\
\hline & R18 & 98.6 & 100.1 & Fast & Absent & Gained & 0 & 0 \\
\hline & R19 & 99.1 & 100.4 & Fast & Absent & Maintained & 0 & 0 \\
\hline & R20 & 98 & 99.2 & Fast & Absent & Gained & 0 & 0 \\
\hline
\end{tabular}

*scoring scale is shown in table 2

A histological study showed that the 3D printed PP mesh worked well with considerable fibro vascular tissue in growth and minor inflammation (Figure 6 A). The PVA mesh showed less fibrovascular tissue infiltration and mild inflammatory reaction with inflammatory cells as seen in the adipose tissue (Figure 6 B). As expected, drug loaded PP and PVA meshes showed little or no inflammatory cells due to the presence of ciprofloxacin $\mathrm{HCl}$ (Figure 6C \& 6D respectively). Reference mesh showed moderate inflammatory reactions, mixed inflammatory cell including macrophages, lymphocytes, plasma cells and neutrophils (Figure 6E). Fibrovascular tissue deposition was less than that with 3D printed PP mesh. 
A

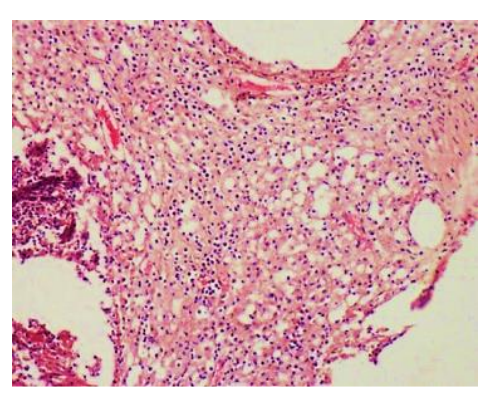

B

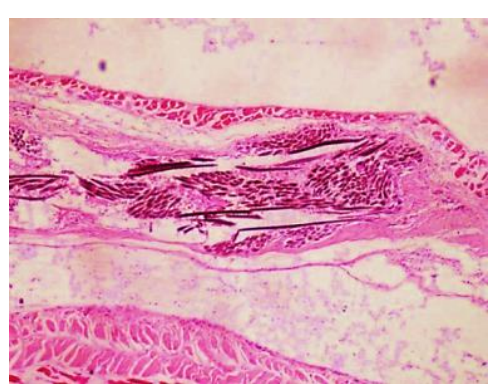

\section{C}

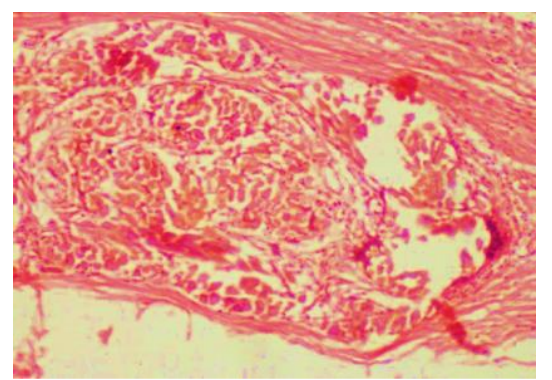

D

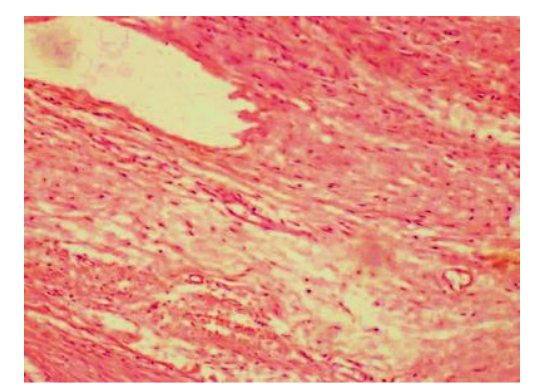

E

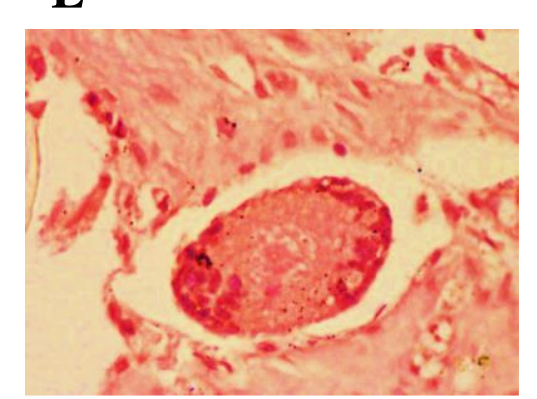

Figure 6. Histological response to implanted meshes (A) PP (B) PVA (C) drug loaded PP (D) drug loaded PVA and (E) reference mesh 


\section{Conclusion}

FDM was effectively used to fabricate hernial meshes with desirable attributes. Both PP and PVA filaments showed adequate drug loading capacity and the drug remained stable through the printing process. The tensile strength of newly formulated meshes was within the acceptable range. In vivo testing using a rabbit model provided no evidence of implant rejection. Compared to the reference mesh, the extent of adhesions to visceral tissue was very low with 3D printed PP meshes and no adhesions developed where PVA meshes were implanted. With present progress in the application of 3D printing in pharmaceutical manufacturing, customized, on-demand and cost effective hernia meshes could work as a substitute to commercially available mesh. However, the implementation of this novel proposal necessitates clinical trials in order to establish if individualized mesh products are superior to existing practices. The researchers' expectation is that $3 \mathrm{D}$ printing can be the next move in improving the quality of hernia repair by reducing costs, improving efficacy and dispensing patient-specific, bio active and pharmacologic agents intended for the individual requirements of the patient.

\section{Acknowledgement}

We would like to thank Syed Faheem Ahmed for assisting in the animal studies.

\section{Conflict of interest}

Authors declares no conflict of interests. 


\section{References}

1. Pascual, G., et al., Behaviour of a new composite mesh for the repair of full-thickness abdominal wall defects in a rabbit model. PloS one, 2013. 8(11): p. e80647.

2. Penttinen, R. and J. Grönroos, Mesh repair of common abdominal hernias: a review on experimental and clinical studies. Hernia, 2008. 12(4): p. 337-344.

3. Klosterhalfen, B., K. Junge, and U. Klinge, The lightweight and large porous mesh concept for hernia repair. Expert review of medical devices, 2005. 2(1): p. 103-117.

4. Rutkow, I.M. and A.W. Robbins, Demographic, classificatory, and socioeconomic aspects of hernia repair in the United States. Surgical Clinics of North America, 1993. 73(3): p. 413-426.

5. Burger, J.W., et al., Long-term follow-up of a randomized controlled trial of suture versus mesh repair of incisional hernia. Annals of surgery, 2004. 240(4): p. 578.

6. Usher, F.C., J. Ochsner, and L. Tuttle Jr, Use of marlex mesh in the repair of incisional hernias. The American Surgeon, 1958. 24(12): p. 969.

7. Massaron, S., et al., Long-term sequelae after 1,311 primary inguinal hernia repairs. Hernia, 2008. 12(1): p. 57-63.

8. Goldstein, H., Selecting the right mesh. Hernia, 1999. 3(1): p. 23-26.

9. Birch, C., The use of prosthetics in pelvic reconstructive surgery. Best Practice \& Research Clinical Obstetrics \& Gynaecology, 2005. 19(6): p. 979-991.

10. Bellón, J.M., et al., Peritoneal regeneration after implant of a composite prosthesis in the abdominal wall. World journal of surgery, 2001. 25(2): p. 147-152.

11. Subbiahdoss, G., et al., Microbial biofilm growth vs. tissue integration:"the race for the surface" experimentally studied. Acta biomaterialia, 2009. 5(5): p. 1399-1404.

12. Labay, C., et al., Antibiotic-loaded polypropylene surgical meshes with suitable biological behaviour by plasma functionalization and polymerization. Biomaterials, 2015. 71: p. 132-144.

13. Gross, B.C., et al., Evaluation of 3D printing and its potential impact on biotechnology and the chemical sciences. 2014, ACS Publications.

14. Feuerbach, T., S. Kock, and M. Thommes, Characterisation of fused deposition modeling 3D printers for pharmaceutical and medical applications. Pharmaceutical development and technology, 2018: p. 1-10.

15. Goyanes, A., et al., Fused-filament 3D printing (3DP) for fabrication of tablets. International journal of pharmaceutics, 2014. 476(1): p. 88-92.

16. Pietrzak, K., A. Isreb, and M.A. Alhnan, A flexible-dose dispenser for immediate and extended release 3D printed tablets. European Journal of Pharmaceutics and Biopharmaceutics, 2015. 96: p. 380-387.

17. Yu, D.G., et al., Novel oral fast-disintegrating drug delivery devices with predefined inner structure fabricated by Three-Dimensional Printing. Journal of Pharmacy and Pharmacology, 2009. 61(3): p. 323-329.

18. Zheng, F. and S.-w. Huang, Advances in Study on Three-dimensional Printing in Pharmaceutics. Chinese Herbal Medicines, 2016. 8(2): p. 121-125.

19. Michalski, M.H. and J.S. Ross, The shape of things to come: 3D printing in medicine. Jama, 2014. 312(21): p. 2213-2214.

20. Colasante, C., et al., Current trends in 3D printing, bioprosthetics, and tissue engineering in plastic and reconstructive surgery. Current Surgery Reports, 2016. 4(2): p. 6. 
21. Weisman, J.A., et al., Antibiotic and chemotherapeutic enhanced three-dimensional printer filaments and constructs for biomedical applications. International journal of nanomedicine, 2015. 10: p. 357.

22. Christian, J.S., The quinolone antibiotics. Primary Care Update for OB/GYNS, 1996. 3(3): p. 87-92.

23. Hedrick, T.L., et al., The appropriate use of antibiotics in surgery: a review of surgical infections. Current problems in surgery, 2007. 44(10): p. 635-675.

24. Habash, M. and G. Reid, Microbial biofilms: their development and significance for medical device - related infections. The Journal of Clinical Pharmacology, 1999. 39(9): p. 887898.

25. USP, C., The United States Pharmacopeia. National Formulary, 2008. 14.

26. Berman, B., 3-D printing: The new industrial revolution. Business horizons, 2012. 55(2): p. 155-162.

27. Silva, W.d., et al., Animal model of chronic abdominal hernia in rabbit. Acta cirurgica brasileira, 2009. 24(4): p. 256-261.

28. Kim, S.W., Y.H. Bae, and T. Okano, Hydrogels: swelling, drug loading, and release. Pharmaceutical research, 1992. 9(3): p. 283-290.

29. Bringman, S., et al., Hernia repair: the search for ideal meshes. Hernia, 2010. 14(1): p. 81-87.

30. Bittner, R., et al., Guidelines for laparoscopic treatment of ventral and incisional abdominal wall hernias (International Endohernia Society (IEHS)-part 1. Surgical endoscopy, 2014. 28(1): p. 2-29.

31. Schreinemacher, M., et al., The need for standardised animal models and scoring systems in assessing mesh biocompatibility. Hernia, 2010. 14(3): p. 335-336.

32. DuBay, D.A., et al., Incisional herniation induces decreased abdominal wall compliance via oblique muscle atrophy and fibrosis. Annals of surgery, 2007. 245(1): p. 140. 33. Franz, M.G., The biology of hernias and the abdominal wall. Hernia, 2006. 10(6): p. 462-471.

34. Dubay, D.A., et al., Fascial fibroblast kinetic activity is increased during abdominal wall repair compared to dermal fibroblasts. Wound repair and regeneration, 2004. 12(5): p. 539-545.

35. Dubay, D.A., et al., The prevention of incisional hernia formation using a delayedrelease polymer of basic fibroblast growth factor. Annals of surgery, 2004. 240(1): p. 179. 